\title{
In vivo post-transcriptional gene silencing of $\alpha-1$ antitrypsin by adeno-associated virus vectors expressing siRNA
}

\author{
Pedro E Cruz ${ }^{1,2}$, Christian Mueller ${ }^{1,2}$, Travis L Cossette ${ }^{2}$, Alexandra Golant ${ }^{3}$, Qiushi Tang ${ }^{1,2}$, Stuart G Beattie ${ }^{1,2}$, \\ Mark Brantly ${ }^{4}$, Martha Campbell-Thompson ${ }^{5}$, Keith S Blomenkamp ${ }^{6,7}$, Jeffrey H Teckman ${ }^{6,7}$ \\ and Terence R Flotte $1,2,4,8,9$
}

$\alpha-1$ Antitrypsin (AAT) deficiency is one of the most common genetic diseases in North America, with a carrier frequency of approximately $4 \%$ in the US population. Homozygosity for the most common mutation (Glu342Lys, $\mathrm{PI}{ }^{\star Z}$ ) leads to the synthesis of a mutant protein, which accumulates and polymerizes within hepatocytes rather than being efficiently secreted. This lack of secretion causes severe serum deficiency predisposing to chronic lung disease. Twelve to fifteen percent of patients with $\mathrm{Pl}^{\star} \mathrm{ZZ}$ also develop liver disease, which can be severe, even in infancy. This is thought to be due to toxic effects of the accumulated mutant Z-AAT within the hepatocyte. Thus, an approach to reduce AAT-deficient liver disease will likely require some mechanism to decrease the amount of Z-AAT within hepatocytes. In this report, we describe studies of small-interfering RNAs (siRNAs) designed to downregulate endogenous AAT within hepatocytes. Three different siRNA sequences were identified and cloned into a recombinant adeno-associated virus (rAAV) backbone, either singly or as a trifunctional (3X) construct. Each had activity independently, but the levels of AAT expression in cell culture models showed the greatest decrease with the $3 X$ construct, resulting in levels that were five-fold lower than controls. The rAAV-3X-siRNA was then packaged into AAV8 capsids and used in vivo to transduce the livers of human Z-AAT overexpressing transgenic mice. Those studies showed a decrease in total human AAT, a clearing of Z-AAT accumulation by immunohistochemistry, and a decrease in monomer Z-AAT within the liver within 3 weeks after vector injection. The rAAV8-3X-siRNA vector may hold promise as a potential therapy for patients with AAT liver disease.

Laboratory Investigation (2007) 87, 893-902; doi:10.1038/labinvest.3700629; published online 25 June 2007

KEYWORDS: $\alpha-1$ antitrypsin; PIZ; siRNA; rAAV

$\alpha-1$ Antitrypsin (AAT) deficiency is a highly prevalent disorder in Northern Europeans and North Americans, with a carrier frequency of up to $4 \% .^{1-6}$ AAT is normally synthesized as a $52 \mathrm{kDa}$ protein secreted from hepatocytes into the serum, and functioning throughout the body as a potent, multifunctional anti-protease. The most common AAT mutation, (Glu342Lys, $\mathrm{PI}^{\star} \mathrm{Z}$ ) causes a well-characterized defect in AAT folding, and a predilection for formation of protein polymers by the loop-sheet insertion mechanism..$^{7-15}$ These properties impede secretion of the Z-AAT protein from hepatocytes. Individuals homozygous for this mutation (designated as $\mathrm{PI}^{\star} \mathrm{ZZ}$ ) have severe deficiency predisposing to chronic lung disease. A significant percentage of patients with $\mathrm{PI}^{\star} \mathrm{ZZ}(12-15 \%)$ also develop liver disease as a result of the accumulated, polymerized protein within liver cells, which can be severe, even in infancy.

Gene therapy strategies for AAT deficiency have mostly focused on gene augmentation as a means to treat AAT lung disease. ${ }^{16-20}$ A variety of different vector approaches have been utilized for this purpose, and AAT expression and secretion can often be accomplished from ectopic sites, such as skeletal muscle. The latter approach is currently under

\footnotetext{
${ }^{1}$ Department of Pediatrics, University of Florida, Gainesville, FL, USA; ${ }^{2}$ Powell Gene Therapy Center, University of Florida, Gainesville, FL, USA; ${ }^{3}$ Department of Pediatrics, Mount Sinai School of Medicine, New York, NY, USA; ${ }^{4}$ Department of Medicine, University of Florida, Gainesville, FL, USA; ${ }^{5}$ Department of Pathology, Immunology and Laboratory Medicine, University of Florida, Gainesville, FL, USA; ${ }^{6}$ Department of Gastroenterology and Hepatology, St Louis University School of Medicine, St Louis, MO, USA; ${ }^{7}$ Department of Pediatrics, St Louis University School of Medicine, St Louis, MO, USA; ${ }^{8}$ Department of Molecular Genetics and Microbiology, University of Florida, Gainesville, FL, USA and ${ }^{9}$ Department of Pediatrics, University of Massachusetts Medical School, Worcester, MA, USA

Correspondence: Dr TR Flotte, MD, Medical School, University of Massachusetts, S1-340, 55 Lake Avenue North, Worcester, MA 1655, USA.

E-mail: Terry.Flotte@umassmed.edu
}

Received 22 December 2006; revised 30 May 2007; accepted 31 May 2007 
examination in phase I clinical trials. ${ }^{21,22}$ A phase I trial of intramuscular injection of a rAAV2-AAT vector has recently been completed, and a phase I trial of a rAAV1-AAT vector has just been initiated.

There have also been some approaches tested in the past animal models that are designed as therapies for AAT liver disease. Such strategies generally seek to use molecular means to downregulate expression of the endogenous mutant ZAAT. One example is the use of a recombinant SV40 vector expressing anti-AAT ribozymes within the liver of the human Z-AAT overexpressing transgenic mouse model. ${ }^{23}$ Since augmentation of the wild-type M-AAT can occur from ectopic sites, these downregulation methods within the liver do not have to necessarily be allele-specific. However, it may be necessary to downregulate Z-AAT within a high percentage of hepatocytes to achieve the desired effect.

The emergence of newer rAAV serotypes, such as rAAV8, that are very efficient for gene delivery in the liver has opened the opportunity for new vector approaches to downregulation of Z-AAT as a therapy for AAT liver disease. ${ }^{19,24-28}$ In this report, a number of small-interfering RNA (siRNA) constructs directed against AAT were expressed from rAAV vectors in vitro and in vivo and their ability to decrease the levels of intracellular Z-AAT within the liver was assessed. A tri-functional (3X) construct emerged from these studies as a potential candidate for future studies of gene therapy of AAT liver disease.

\section{METHODS}

\section{siRNAs Design Strategy}

Ambion's (Ambion Inc., 2130 Woodward St Austin, TX, USA) siRNA design tool allowed for selection of possible target areas from the human AAT (SERPINA1) mRNA coding region. This tool is based on the article written by Tuschl et al. ${ }^{29-31}$ Three siRNA sequences were selected (Table 1) and further analyzed for their efficiency against AAT expression. One siRNA (AAT1) targets the $5^{\prime}$-end of the coding region. The second siRNA (AAT33) targets the middle, and the third siRNA (AAT55) is specific for the $3^{\prime}$-end of the human AAT open reading frame. The sequences of all siRNAs are specific only to the human AAT gene as indicated by a BLAST search. The Ambion's negative control (NC) siRNA was used as a negative control.

Table 1 Sense DNA sequence for siRNA

\begin{tabular}{lll}
\hline siRNA sequence & siRNA name & hAAT gene targeted exon \\
\hline GACAGATACATCCCACCAT & AAT1 & Exon 2 \\
AGGCAAATGGGAGAGACCC & AAT33 & Exon 3 \\
CCTATGATCTGAAGAGCGT & AAT55 & Exon 4 \\
ACTACCGTTGTATAGGTG & NC & Negative control siRNA \\
\hline
\end{tabular}

\section{siRNA-Expressing Constructs}

All siRNAs were cloned into the BamHI and HindIII sites of the Ambion's pSilencer-2 plasmid using the following complementary oligos having BamHI and HindIII sticky ends: AAT1-F: GATCCCGACAGATACATCCCACCATTTCAAGAG AATGGTGGGATGTATCTGTCTTTTTTGGAAA; AAT1-R: AGCTTTTCCAAAAAAGACAGATACATCCCACCATTCTCTT GAAATGGTGGGATGTATCTGTCGG; AAT33-F: GATCCCA GGCAAATGGGAGAGACCCTTCAAGAGAGGGTCTCTCCC ATTTGCCTTTTTTTG; AAT33-R: AGCTTTTCCAAAAAAA GGCAAATGGGAGAGACCCTCTCTTGAAGGGTCTCTCCC ATTTGCCTGGGAAA; AAT55-F: GATCCCGCCTATGATCT GAAGAGCGTTTCAAGAGAACGCTCTTCAGATCATAGGT TTTTTGGAAA; AAT55-R: AGCTTTTCCAAAAAACCTATG ATCTGAAGAGCGTTCTCTTGAAACGCTCTTCAGATCATA GGCGG. The plasmid allows transcription of siRNA as a hairpin by using the U6 RNA polymerase III promoter. Transcripts of siRNA form RNA hairpins that are processed by the DICER enzyme to yield short double-stranded RNA molecules (siRNA). The siRNA expression cassette from the pSilencer-2 was subcloned into the proviral AAV plasmid. Proviral AAV plasmid was used to make recombinant AAV virus expressing siRNA. All three siRNAs against AAT were consolidated into a single AAV expression vector. This allowed a single plasmid to carry all siRNAs and save in the cost of producing AAV virus. Two versions of the AAV siRNA vector exist having neomycin (neo) or green fluorescent protein (GFP) marker under the control of HSV-1 TK promoter.

\section{rAAV8 Packaging and Purification}

Serotype 2 rAAV and rAAV pseudotyped into capsid from serotype 8 were produced by the Vector Core Laboratory, Powell Gene Therapy Center, University of Florida as described before. ${ }^{19,32}$

\section{Human Cell Lines}

The HEK-293 cell line (ATCC no. CRL-1573, Manassas, VA, USA) and the HepG2 liver cell line (ATCC no. HB-8065) were used to test siRNA efficacy against AAT expression. HepG2 cell lines constitutively express high levels of AAT.

\section{Transient Transfection, Cell Culture and FACS Analysis}

All experiments were performed using six-well cell culture plates (Falcon 353046, Becton Dickinson, Franklin Lakes, NJ, USA). Lipofectamine 2000 cationic lipid (Invitrogen Corp., Carlsbad, CA, USA) was used to co-transfect HEK-293 cells with the M-AAT or Z-AAT expression plasmids and siRNA expression plasmids. HepG2 cells were transfected with siRNA plasmid using the GeneJammer reagent (Stratagene, La Jolla, CA, USA). HepG2 were transduced with $1 \mathrm{~K}$ MOI of rAAV-2 expressing siRNA and $1 \mathrm{MOI}$ of adenovirus-5 as helper virus.

Cells lines were transfected with plasmid expressing siRNA and GFP marker and sorted using FACSort (FACS Vantage 
SE Turbosort with DiVa, BD Bioscience, San Jose, CA, USA) performed by the University of Florida-Interdisciplinary Center for Biotechnology Research-Flow Cytometry Laboratory. Lysate of FACSort-sorted cells were analyzed by ELISA.

\section{ELISA}

hAAT protein levels were detected by ELISA as described previously. ${ }^{19,33}$ High binding extra, 96-well plate (Immulon 4, Dynatech, catalogue no. 3855) were coated with $100 \mu \mathrm{l}$ of goat anti-hAAT (1:200 diluted; MP Pharmaceuticals no. $55111)$ in Voller's buffer overnight at $4^{\circ} \mathrm{C}$. Duplicate standard curves (hAAT; Sigma, catalogue no. A-9024) and serially diluted unknown samples were incubated in the plate at $37^{\circ} \mathrm{C}$ for $1 \mathrm{~h}$. After blocking with 3\% bovine serum albumin (BSA Sigma catalogue no. B4287), a second antibody, rabbit antihAAT (1:1000 diluted, Sigma catalogue no. A0409) was reacted with the captured antigen at $37^{\circ} \mathrm{C}$ for $1 \mathrm{~h}$. A third antibody, goat anti-rabbit IgG conjugated with peroxidase (1:5000 diluted; Sigma catalogue no. A0545) was incubated at $37^{\circ} \mathrm{C}$ for $1 \mathrm{~h}$. The plate was washed with phosphate-buffered saline (PBS)-Tween 20 between reactions. After reaction with TMB peroxidase (KPL Kit, catalogue no. 50-76-00) substrate, stop reaction by adding $1 \mathrm{M} \mathrm{H}_{3} \mathrm{PO}_{4}$ (Fisher, catalogue no. A242-500). Plates were read at $450 \mathrm{~nm}$ on a VersaMax microplate reader (Molecular Devices).

\section{Portal Vein Injection of rAAV8 Vectors In Z-AAT Transgenic Mice}

PiZ-transgenic mice have been described previously. ${ }^{34-37}$ Injection has been described previously. ${ }^{19}$ Work is carried out completely under a sterilized laminar flow hood in SPF animal facility. A portable rodent isoflourane machine is used at $3 \%$ mixture. The preparation time for the surgery is approximately $1 \mathrm{~h}$. All aseptic rodent surgery guidelines are strictly followed. The mouse is first identified with an ear tag, placed into induction chamber and initially administered 5\% isoflourane, which is then dropped down to $2-3 \%$ once the proper plane of anesthesia is achieved. A non-steroidal ophthalmic ointment is applied over eyes of mouse to keep moist. The mouse is placed in dorsal recumbancy with the anesthesia mask over its head. A depilatory cream is applied onto the surgical site and is let set for approximately 1-2 min. After completely removing all depilatory substance from the surgical site, it is prepped with iodine and finally wiped away with $70 \%$ isopropyl alcohol. The initial incision of the dermal layer is started and after dermal layer is cut, an incision of the visceral layer is performed. Sterile $2 \times 2^{\prime}$ gauze moistened with $1 \times$ PBS is placed immediately parallel to the incision. Sterile cotton-tipped applicators (also moistened with $1 \times$ PBS) are used to gently lift out the intestines onto the sterile gauze. Once the intestines have been successfully moved, the chest is spread. Chest spreaders are placed on each side of the incision to expose the area around the portal vein. Once the chest is exposed, sterile cotton tipped applicators are used to lift up the fatty area under the first lobe of the liver. Under the lobe the portal vein is clearly visible. A $31 \mathrm{~g} 3 / 10 \mathrm{ml}$ insulin syringe is used to inject a $100 \mu \mathrm{l}$ volume of virus/PBS mixture into the portal vein. At this point the needle is at approximately a $10^{\circ}$ angle to the vein. The needle is carefully placed into the vein and the plunger on the syringe is depressed to inject the virus. As the needle is pulled out, a cotton tipped applicator is quickly placed over the injection site. Compression of this vein to stop bleeding can sometimes take up to $2-3 \mathrm{~min}$. The applicator is slowly removed from the portal vein and the area is watched closely for any signs of bleeding. If bleeding takes place new applicators are placed over the injection site. Once the bleeding stops, the intestines are placed back into abdominal cavity using applicators moistened with $1 \times$ PBS. The chest spreaders are removed from the mouse and intestines are re-hydrated with a pipette and 100-200 $\mu \mathrm{l}$ of $1 \times$ PBS. The visceral layer is sewn with 5-0 Vicryl suture using a simple continuous loop stitch with interrupts of square knots on the ends. The dermal layer is then sewn using 5-0 Ethilon suture. The mouse is then carefully placed in dorsal recumbence inside of a clean cage on top of a heating pad set on low and the animal is then monitored closely until it recovers completely from anesthesia. Post-surgical guidelines are followed according to IACUC policy.

\section{Histology, Histochemistry and Immunohistochemistry on rAAV8-Injected Mice}

Histology was performed on organs from all mice harvested at day 14 as a final experimental end point. For determination of histological changes, liver samples were fixed in $10 \%$ neutral-buffered formalin (Fisher Scientific), and embedded in paraffin. Sections $(5 \mu \mathrm{m})$ were stained with hematoxylin and eosin and periodic acid-Schiff (PAS) with or without diatase treatment. Immunohistochemistry was performed using rabbit anti-human AAT (Research Diagnostics Incorporated) or anti-GFP (Abcam) as described previously. ${ }^{19}$

\section{Histological Analysis}

For computerized quantification of histochemistry and immunohistochemistry slides, 10 random fields at $\times 10$ were photographed from the liver sections for each mouse. The pictures where then analyzed using MetaMorph 6.3r7 software. Positive pixels were selectively detected and uniformly displayed with an orange pixel overlay using the threshold function. The threshold area corresponding to positivestained area and was measured with the $<$ region measurement $>$ function.

\section{Monomer-Polymer AAT Analysis on Liver Extracts}

Analysis of Z-AAT protein monomer and polymer content in the liver has been described previously. ${ }^{38-40}$ For soluble/insoluble protein separation (Figure $7 \mathrm{a}$ ), $10 \mathrm{mg}$ of whole liver was added to $2 \mathrm{ml}$ buffer at $4{ }^{\circ} \mathrm{C}(50 \mathrm{mmol} / \mathrm{l}$ Tris- $\mathrm{HCl}(\mathrm{pH}$ 8.0), $150 \mathrm{mmol} / \mathrm{l} \mathrm{NaCl}, 5 \mathrm{mmol} / \mathrm{l} \mathrm{KCl}, 5 \mathrm{mmol} / \mathrm{l} \mathrm{MgCl} 2,0.5 \%$ Triton X-100 and $80 \mu \mathrm{l}$ Complete ${ }^{\mathbb{R}}$ protease inhibitor stock). 
The tissue was homogenized in a prechilled Dounce homogenizer for 30 repetitions, then vortexed vigorously. A 1-ml aliquot was passed through a 28 -gauge needle 10 times. The total protein concentration of the sample was determined, and a 5- $\mu$ g total liver protein sample was aliquoted and centrifuged at $10000 \mathrm{~g}$ for $30 \mathrm{~min}$ at $4^{\circ} \mathrm{C}$. Supernatant (soluble (S) fraction) was immediately removed into fresh tubes; extreme care was taken to avoid disturbing the pellet (insoluble (I) fraction). The insoluble polymers pellet (I fraction) was denatured and solubilized via addition of $10 \mu \mathrm{l}$ chilled cell lysis buffer ( $1 \%$ Triton X-100, $0.05 \%$ deoxycholate, $10 \mathrm{mmol} / \mathrm{l}$ EDTA in PBS), vortexed for $30 \mathrm{~s}$, sonicated on ice for $10 \mathrm{~min}$ and vortexed. To each soluble and insoluble sample $2.5 \times$ sample buffer $(50 \% 5 \times$ sample buffer (5\% sodium dodecyl sulfate, $50 \%$ glycerol, $0.5 \mathrm{~mol} / \mathrm{l}$ Tris (pH 6.8)), 10\% $\beta$-mercaptoethanol, $40 \% \mathrm{ddH}_{2} \mathrm{O}$ ) was added at a volume of $50 \%$ of the sample volume. Samples were boiled and loaded for sodium dodecyl sulfate-polyacrylamide gel electrophoresis (SDS-PAGE); equal amounts of total liver protein were loaded per soluble-insoluble pair in quantitative experiments. Densitometry was performed using Scion Image software (Scion Corporation, Frederick, MD, USA).

\section{Serum Chemistry}

All serum samples were analyzed by the NExCT Clinical Chemistry Analyzer (Alfa Wassermann Diagnostic Technologies, LLC, 4 Henderson Dr, West Caldwell, NJ, USA). Serum was analyzed for alanine aminotransferase (ALT) and aspartate aminotransferase (AST). Manufacturer (Alfa Wassermann Diagnostic Technologies, LLC, 4 Henderson Dr, West Caldwell, NJ, USA) methodology was followed to analyze the serum of mice collected at killing (day 14).

\section{Statistical Analysis}

All data in Figures 1a, b, 2, 4-6, 7b and 8 were compared using an unequal variance or a paired two-tail Student's $t$-test (Jandel SigmaStat v2, Chicago, IL, USA), and error bars are reported as standard error of the mean. Data were considered statistically significant when $P \leq 0.05$ and all $P$-values $\leq 0.05$ are reported as ${ }^{\star} P \leq 0.05$. Data in Figure 1c were subjected to
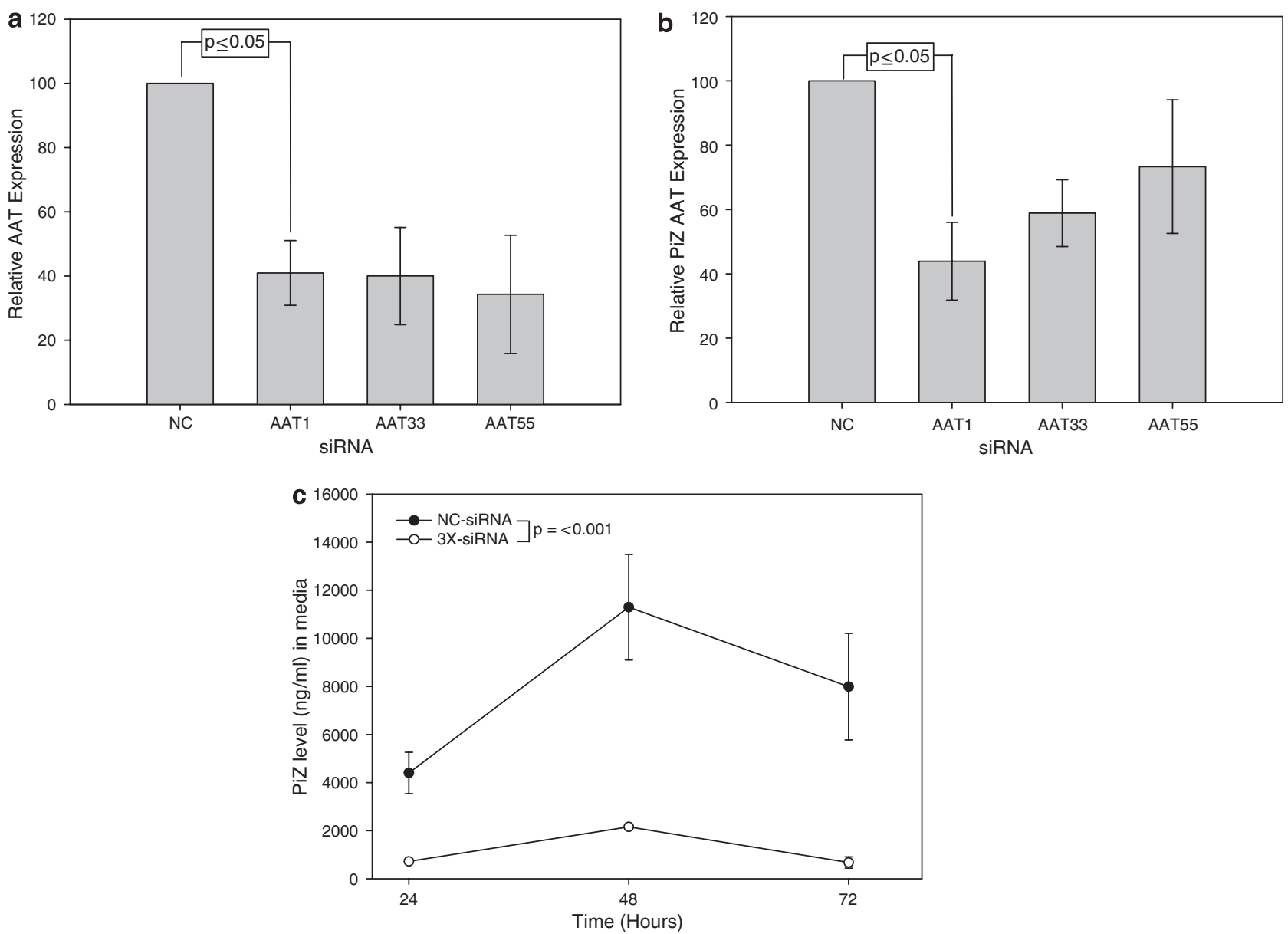

Figure 1 Downregulation of human PiM and PiZ AAT expression by siRNA. HEK-293 cells were co-transfected with hAAT (a) or PiZ (b) and siRNA plasmid. After $48 \mathrm{~h}$ of transfection media was collected and AAT level measure by ELISA. (c) HEK-293 cells were co-transfected with PiZ and a trifunctional (3X) plasmid composed of the three siRNAs tested in (a) and (b). Culture media was harvested at three time points and was analyzed for PiZ AAT levels. In (a) and (b), $n=2$ wells per siRNA on a six-well plate. (c) $n=4$ wells per siRNA on six-well plates. Data are expressed as the mean \pm s.e.m. 

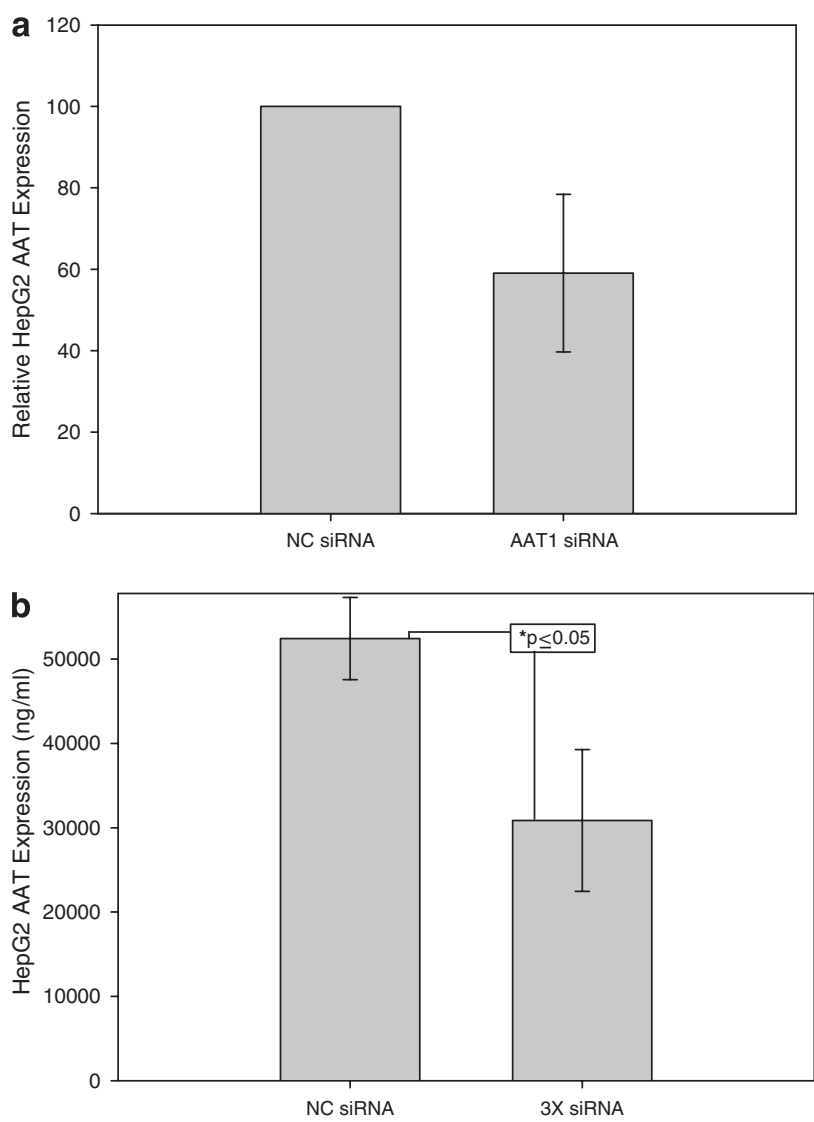

Figure 2 Downregulation of human AAT expression by siRNA. (a) HepG2 cells were transduced by rAAV2-U6-AAT1-siRNA or rAAV2-U6-NC-siRNA using an MOI of 1000. After $72 \mathrm{~h}$ media was collected and AAT level was measured by ELISA. (b) HepG2 cells were co-transfected with pTR2-U6-NC EGFP-TK (negative control) or PTR2-U6-AAT1-U6-AAT55-U6-AAT33-EGFP-TK (tri-functional) plasmids. At $72 \mathrm{~h}$ cells were FACSorted and cells' lysate were analyzed for AAT level measured by ELISA. (a) $n=2$ wells per rAAV-siRNA on a six-well plate; (b) $n=3$ wells per plasmid-siRNA on a six-well plate. Data are expressed as the mean \pm s.e.m.

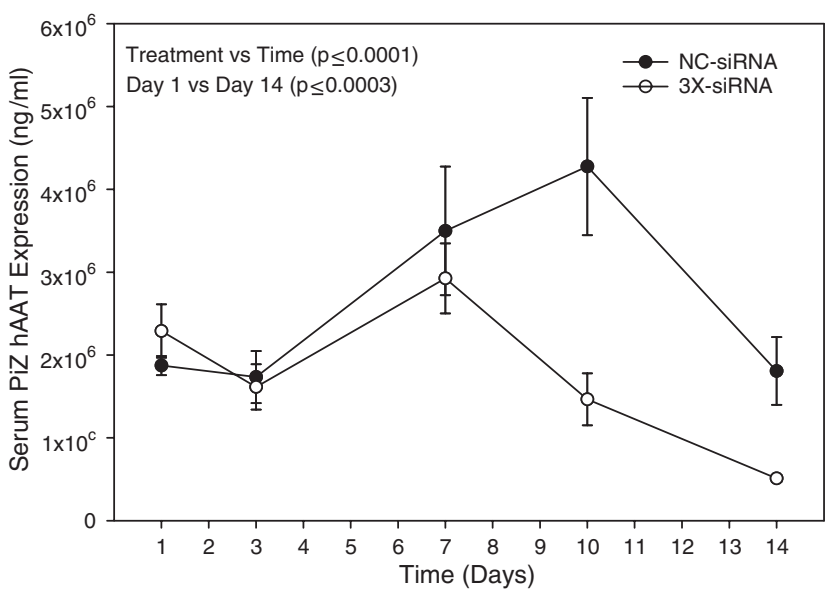

Figure 3 In vivo downregulation of human AAT expression by siRNA. PiZ-transgenic mice were injected with $1 \times 10^{11}$ vector/genomes of rAAV8expressing siRNAs against hAAT or negative control via the portal vein. Serums were collected at several time points post injection and were analyzed for AAT levels by ELISA. Data are expressed as the mean \pm s.e.m. $(n=4)$. a two-way repeated measures ANOVA (Jandel SigmaStat v2). Data in Figure 3 were compared by repeated measure analysis of eight subjects with four in each group using the Greenhouse-Geisser and was conducted by using PROC GLM in SAS. Greenhouse-Geisser test shows that the time and the interaction between time and treatment are both highly significant $(P<0.0001$ and $P=0.002)$.

\section{RESULTS \\ Downregulation of Z-AAT in Transiently Transfected Cell Lines}

The rAAV-siRNA constructs described in Tables 1 and 2 were initially co-transfected into HEK-293 cells with either M-AAT or Z-AAT to examine the efficiency of downregulation. In the case of M-AAT, culture media levels decreased to approximately $40 \%$ of baseline with each of the three siRNAs (Figure 1a). With ZAAT, the reduction was also to approximately $40 \%$ of baseline with the AAT1 siRNA, but was less extensive with either of the two other siRNAs alone (Figure 1b). However, when all three active siRNAs were combined in a single construct (3X) the effect in transient co-transfection with Z-AAT in the cell line was much greater. The maximal effect, a five-fold reduction, was seen at day 2 post-transfection (Figure 1c).

\section{Downregulation of Stable Endogenous Human AAT Expression in HepG2 Cells}

To determine whether the effects of the rAAV-siRNAs were generalizable to a stably expressed, endogenous AAT allele, we transduced HepG2 cells with a rAAV vector expressing NC-siRNA or the AAT1 siRNA. Despite the fact that these

Table 2 siRNA plasmids used for transfection or for rAAV production

\begin{tabular}{ll}
\hline Plasmid name & Description \\
\hline pSilencer2-U6-NC & Expressing negative control \\
& siRNA as a hairpin \\
pSilencer2-U6-AAT1 & Expressing siRNA against 5' \\
& of the hAAT mRNA \\
pSilencer2-U6-AAT33 & Expressing siRNA against middle \\
pSilencer2-U6-AAT55 & of the hAAT mRNA \\
& Expressing siRNA against 3' \\
pTR2-U6-NC-TK-Neo & of the hAAT mRNA \\
pTR2-U6-AAT1-TK-Neo & rAAV proviral plasmid \\
pTR2-U6-AAT33-TK-Neo & expressing siRNA mRNA \\
pTR2-U6-AAT55-TK-Neo & rAAV proviral plasmid expressing siRNA \\
pTR2-U6-3X-TK-Neo & rAAV proviral plasmid expressing siRNA \\
pTR2-U6-NC-EGFP-TK & rAAV proviral plasmid expressing siRNA \\
pTR2-U6-3X-EGFP-TK & Expressing all siRNA against hAAT mRNA \\
& rAAV proviral carrying EGFP marker \\
& rAAV proviral carrying EGFP marker
\end{tabular}


studies were carried out with a monofunctional construct, human AAT expression was reduced by nearly 50\% (Figure 2a). Because transduction and transfection efficiency of these cell lines is relatively low, we used a new construct with the GFP reporter gene as well as the siRNA so that cells transfected could be sorted for GFP expression. The GFP-expressing subset was then used to examine downregulation by AAT allele (Figure 2b). This provided an important indication of a positive effect on target RNA transcribed from a stably expressing, endogenous AAT allele.

\section{In Vivo Downregulation of Human Z-AAT Expression}

To determine whether Z-AAT could be reduced in an intact liver, cohorts of Z-AAT transgenic mice were transduced with $1 \times 10^{11}$ vector genomes of the rAAV8-3X-siRNA construct. Measurement of circulating serum levels of human Z-AAT showed a significant reduction (approximately two- to threefold) (Figure 3) as compared with levels observed with a control siRNA vector. Repeated measure analysis determined that the time and the interaction between time and treatment are both highly significant $(P \leq 0.0001$ and $P \leq 0.002)$. Further analysis using a two-way ANOVA showed that the difference of the two treatments at day 1 was statistically different $(P \leq 0.0003)$ from the difference of the two treatments at day 14. This indicates a significant reduction in the PiZ AAT levels at day 14 in the $3 \mathrm{X}$-siRNA group.

Next, we sought to determine whether AAT accumulation would be cleared after hepatic delivery of the rAAV83X-siRNA construct. To determine whether the transduction efficiency was equivalent between the active rAAV83X-siRNA and the rAAV8-NC-siRNA, immunostaining was performed on transduced livers for neomycin phospho-
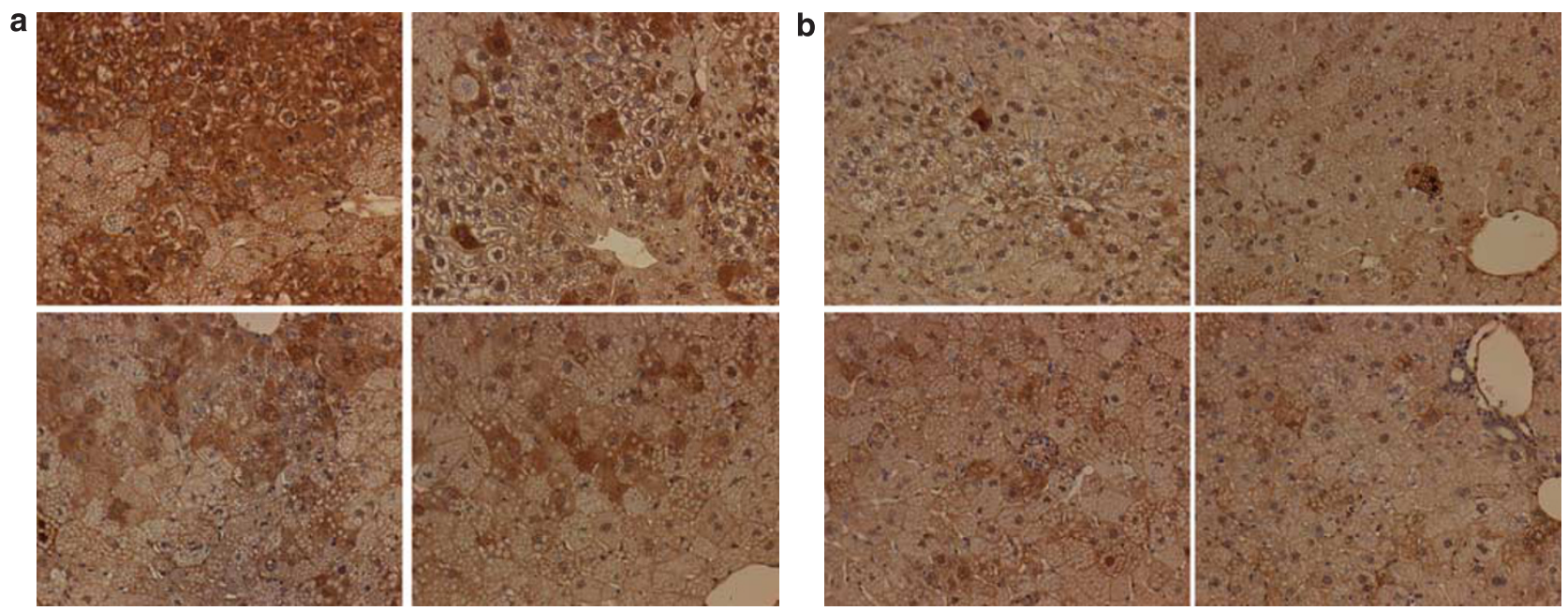

NC-siRNA
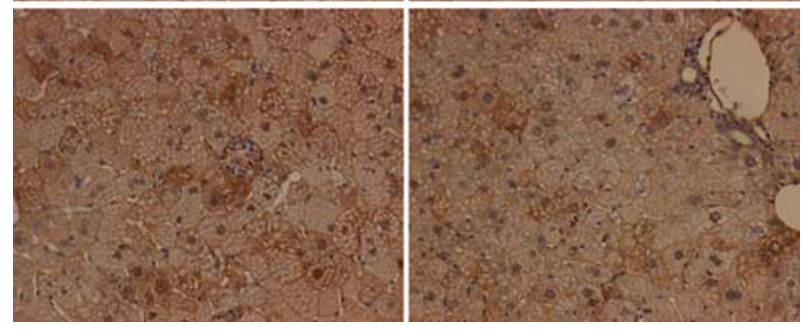

3X-siRNA

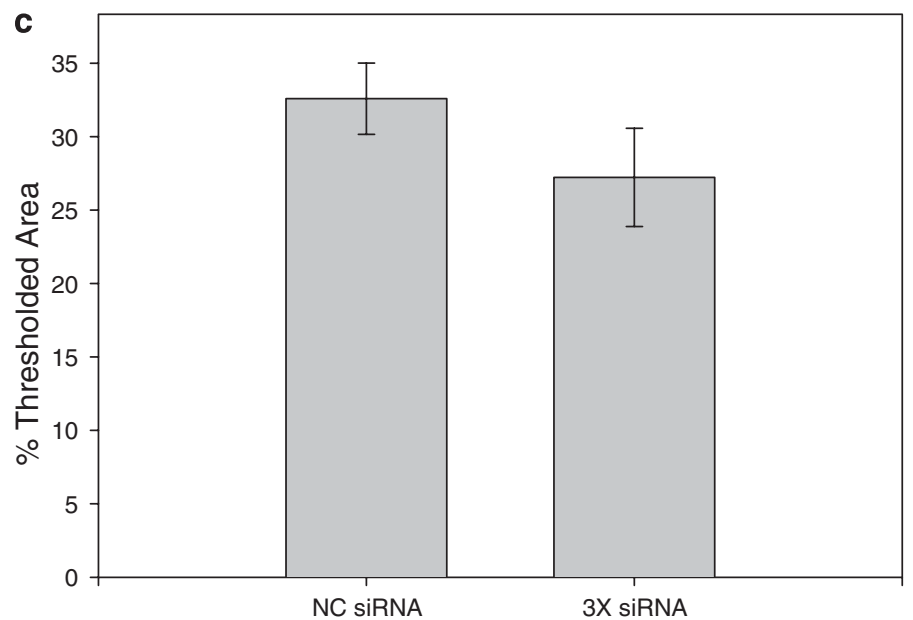

Figure 4 Detection of rAAV8 liver transduction by neomycin staining on PiZ-transgenic mice 14 days post-rAAV8 delivery. Mice were either injected with rAAV8-NC-siRNA or AAV8-3X-siRNA, both of which express neomycin. (a) Representative neomycin-stained liver sections for mice injected with $1 \times 10^{11}$ vector/genomes of rAAV8-NC-siRNA. (b) Representative neomycin-stained liver sections for mice injected with $1 \times 10^{11}$ vector/genomes of rAAV8-3X-siRNA. (c) Quantification of neomycin-stained areas were performed using MetaMorph Software, sections for each rAAV8-siRNA transduced livers were analyzed. Thresholded area represents the area on the section that is within the parameters considered to be positive staining. Results are expressed as the mean thresholded area for four different livers for each group \pm s.e.m. 
transferase, expressed from neo gene cassettes inside both vectors. Fortunately, the neo cassette was small enough to fit inside the packaging capacity of rAAV8 along with the $3 \mathrm{X}$ siRNA-expressing cassette. Immunohistochemistry indicated equivalent staining for transgene expression in both groups of mice (Figure 4$)$. In contrast, hAAT- $\mathrm{Pi}^{\star} \mathrm{Z}$ protein was detectable in the rAAV8-NC-siRNA, and this intracellular Z-AAT signal was significantly cleared in rAAV8-3X-siRNA (Figure 5). The clearing was somewhat heterogeneic, but overall was markedly different between hAAT- $\mathrm{Pi}^{\star} \mathrm{Z}$ livers treated with the active siRNA construct and the control. Staining for PAS-positive material after diastase digestion was similarly intense in the controlled, and cleared in a patchy distribution in the animals treated with the active 3X-siRNA vector (Figure 6).

\section{Selective Decrease in Monomer vs Polymer Z-AAT}

Z-AAT is appropriately transcribed and translated, but when the nascent polypeptide enters the ER lumen it undergoes abnormal folding and very few molecules reach a secretioncompetent conformation. Some of the individual (monomeric) Z-AAT molecules bind together into long, polymeric chains and reside in the ER of the cell in a conformation with a very long biological half-life. To determine whether the effects of the rAAV8-3X-siRNA were on the monomer pool of Z-AAT $v s$ the polymer pool, we analyzed liver samples using a previously published assay. First, the Z-AAT polymers are isolated from the monomers in the liver lysates under non-denaturing conditions and then the separate polymer and monomer fractions are denatured and compared by quantitative immunoblot. The denaturation step reduces the polymers to monomers so the bands can be compared at the same molecular weight. ${ }^{38,40}$ On the basis of the results, there was a marked decrease in hAAT monomer after 2 weeks, while polymer AAT remained relatively stable over this period (Figure 7). This result is consistent with the prediction that rAAV8-3X-siRNA would interrupt the de novo production of Z-AAT and therefore reduce the pool of newly synthesized
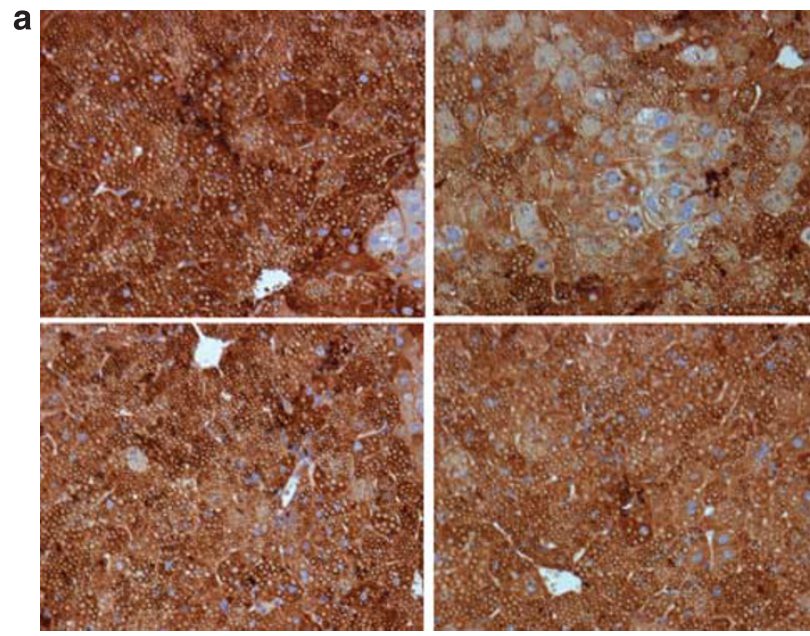

NC-siRNA
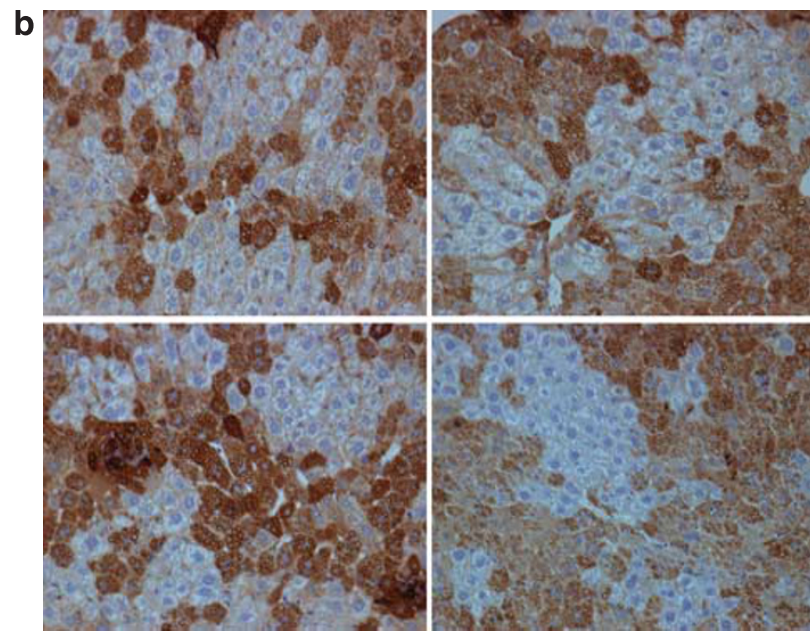

3X-siRNA

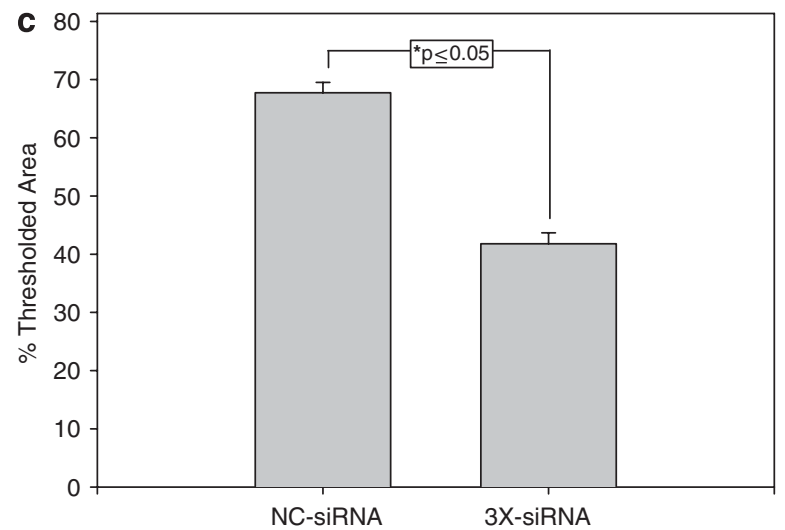

Figure 5 Liver hAAT histology results for PiZ-transgenic mice transduced with AAV8-NC-siRNA or AAV8-3X-siRNA, 14 days post-rAAV8 delivery. (a) Representative hAAT-stained liver sections for mice injected with $1 \times 10^{11}$ vector/genomes of rAAV8-NC-siRNA. (b) Representative hAAT-stained liver sections for mice injected with $1 \times 10^{11}$ vector/genomes of rAAV8-3X-siRNA. (c) Quantification of hAAT-stained areas were performed using MetaMorph Software, sections for each rAAV8-siRNA transduced livers were analyzed. Thresholded area represents the area on the section that is within the parameters considered to be positive staining. Results are expressed as the mean thresholded area for four different livers for each group \pm s.e.m. 

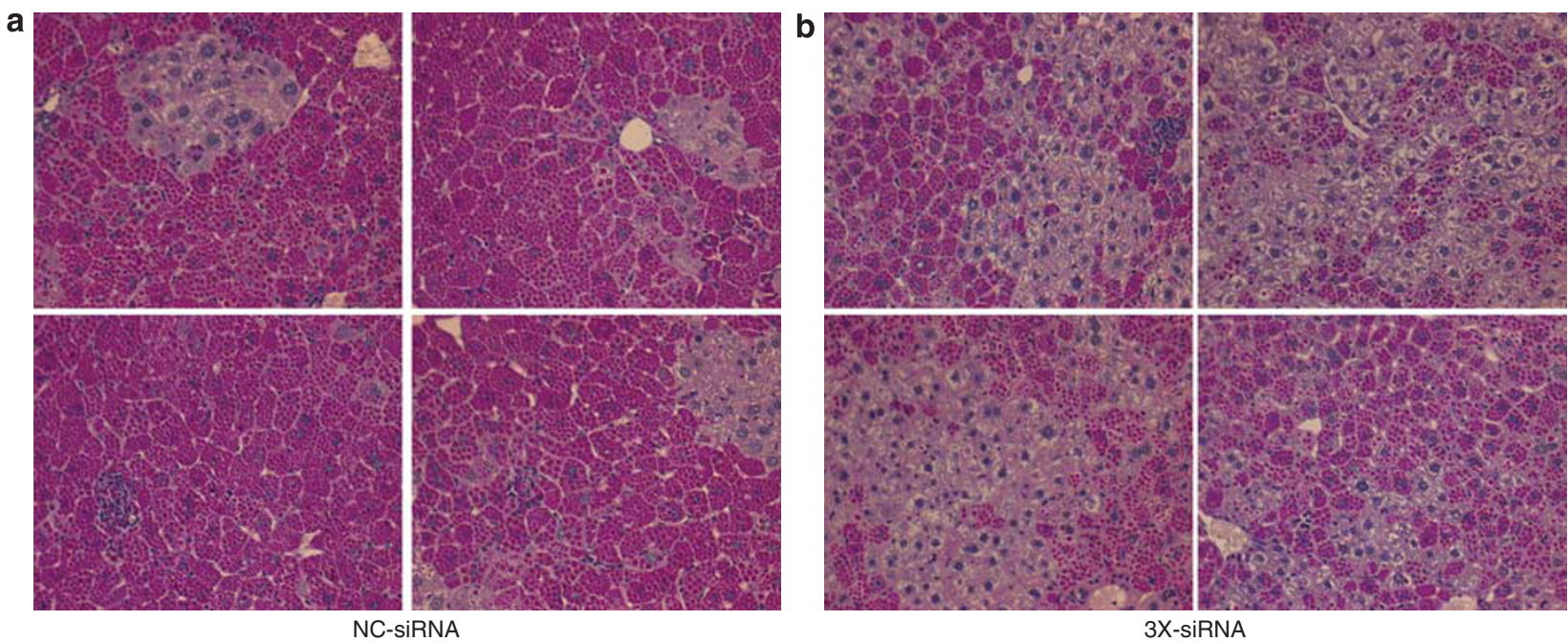

3X-siRNA

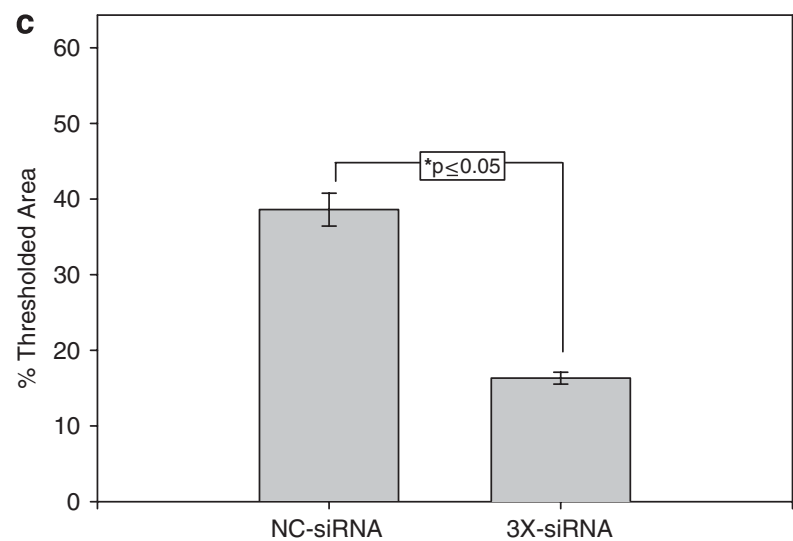

Figure 6 Liver PAS-D histology results for PiZ-transgenic mice transduced with AAV8-NC-siRNA or AAV8-3X-siRNA, 14 days post-rAAV8 delivery. (a) Representative PAS-D stained liver sections for mice injected with $1 \times 10^{11}$ vector/genomes of rAAV8-NC-siRNA. (b) Representative PAS-D stained liver sections for mice injected with $1 \times 10^{11}$ vector/genomes of rAAV8-3X-siRNA. (c) Quantification of PAS-D stained areas were performed using MetaMorph Software, sections for each rAAV8-siRNA transduced livers were analyzed. Thresholded area represents the area on the section that is within the parameters considered to be positive staining. Results are expressed as the mean threshold area for four different livers for each group \pm s.e.m.

monomer, but not affect the previously accumulated and exceedingly stable Z-AAT protein polymer. The ability of this decrease in Z-AAT production to eventually result in a decreased accumulation of polymer Z-AAT over a long period of time was not tested in these experiments.

\section{Serum Chemistry}

To determine whether the transduction of the hepatocytes by rAAV8 or the transcription of the siRNA within the hepatocytes induced any hepatocellular toxicity, the serum levels of ALT and AST were measured 14 days after treatment (Figure 8). Both groups of siRNA-treated mice show serum levels within normal range for both enzymes (ALT normal range: $28-184 \mathrm{U} / \mathrm{l}$ and AST normal range: 55-255 U/l). Mice treated with $3 \mathrm{X}$-siRNA showed a reduction in the serum levels of both enzymes when compared with control siRNAtreated mice. Only the reduction AST levels were statistically significant $(P \leq 0.05)$.

\section{DISCUSSION}

The results presented here clearly demonstrate the biological activity of anti-AAT siRNAs expressed from an U6 promoter in the context of a rAAV vector construct. The ability to downregulate endogenous M-AAT or Z-AAT was demonstrated in both cell culture models and in vivo after portal vein injection of a rAAV8-pseudotyped version of the trifunctional 3X-siRNA vector. The biological effects included a decrease in the amount of Z-AAT secreted from cells and a decrease in the intracellular accumulation of Z-AAT within the liver of transgenic mice. The latter effect was primarily due to a reduction of monomer forms of the mutant protein. A high percentage of hepatocytes showed immunohistochemical evidence of a decrease in intracellular Z-AAT, which is consistent with earlier studies by a number of groups, indicating that rAAV8-pseudotyped vectors are quite efficient for transduction of murine liver. ${ }^{19,28,41,42}$ In fact, the inability of the original rAAV2-based vectors 
a
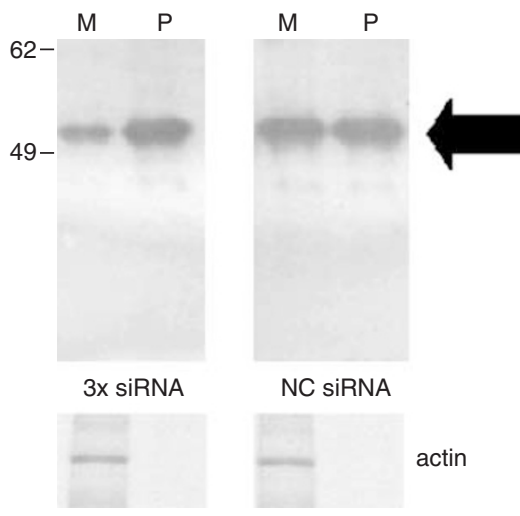

NC siRNA
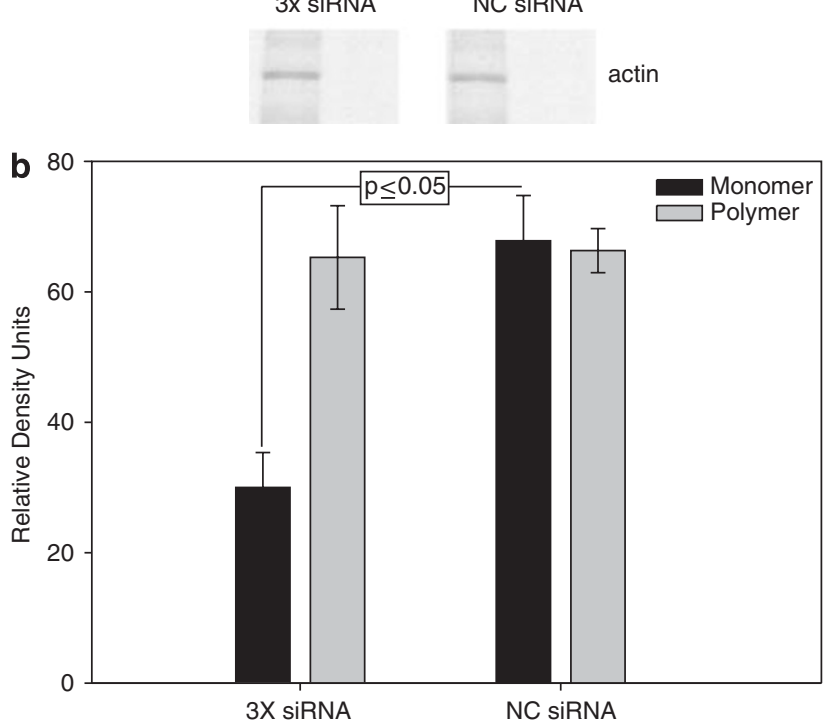

Figure 7 Hepatocytes $\mathrm{PiZ}$ monomer vs polymer densitometry analysis at 14 days post-rAAV8 delivery. (a) Immunoblot for a1AT after the monomer (M) and polymer (P) separation protocol for liver lysates from mice treated with 3XsiRNA or NC-siRNA. Here 49 and $62 \mathrm{kDa}$ markers are shown, arrow is $52 \mathrm{kDa}$ a1 AT mutant $\mathrm{Z}$. Actin immunoblot loading control shown. (Note: the monomer lane contains all hepatocyte protein species, polymer lane is only a1AT polymer extracted from the monomer lane, as demonstrated previously, and is therefore negative for actin.) (b) The immunoblot was scanned and the intensity of each band was determined using Scion Image Software and expressed as relative density units. The results are expressed as mean \pm s.e.m. $(n=4)$.

to transduce stably a high percentage of hepatocytes was a fundamental limitation preventing application of molecular downregulators to Z-AAT-related pathology in the past. ${ }^{43,44}$

The decrease in Z-AAT in both in vitro and in vivo assays suggested that rAAV-3X-siRNA may be potent enough to decrease Z-AAT protein expression to a level similar to or lower than that observed in heterozygous $\mathrm{PI}^{\star} \mathrm{MZ}$ individuals. These individuals do not experience severe AAT liver disease as observed in homozygous $\mathrm{PI}^{\star} \mathrm{ZZ}$ patients. There is some indication that $\mathrm{PI}^{\star} \mathrm{MZ}$ individuals might have an increased risk of chronic liver disease later in life, but the pediatric liver disease typical of $\mathrm{PI}^{*} \mathrm{ZZ}$ patients is generally not seen. ${ }^{3}$ This suggests that clinical benefit might be derived from decreases of Z-AAT of the magnitude observed in this study.
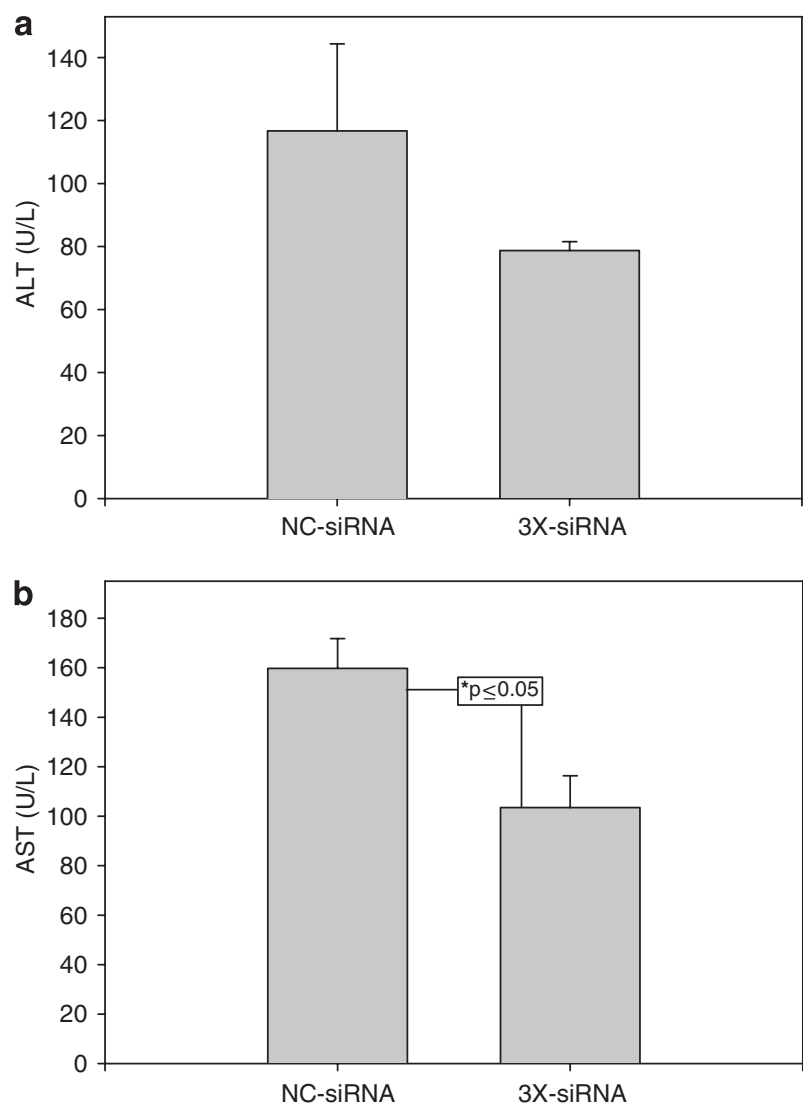

Figure 8 Serum levels for ALT and AST at 14 days post-rAAV8 delivery. (a) Serum levels of ALT. (b) Serum levels of AST. All serum samples were analyzed by the NEXCT Clinical Chemistry Analyzer (Alfa Wassermann Diagnostic Technologies, LLC). The results are expressed as mean \pm s.e.m. $(n=4)$.

These studies were limited in a number of ways. In vivo studies were relatively short term. This did not allow sufficient time to test the ability of this vector approach to ultimate lead to a decrease in intrahepatic Z-AAT polymers. It also limited the ability to examine any salutary effects that the 3X-siRNA might have on the rather subtle liver pathology present in the Z-AAT transgenic mice. Larger studies in the future with longer time courses will be planned to examine the downstream effects of reduced Z-AAT production and to determine if reductions in Z-AAT polymer accumulation will result. $^{6}$

Recent studies by Kay et $a l^{45}$ suggest that overexpression of siRNA in mouse liver could produce hepatotoxicity, perhaps due to interference with the endogenous microRNA mechanisms operative within these cells. To identify any potential hepatocellular toxicity, two markers of hepatocellular injury were measured in the rAAV8-siRNA-treated mice in our study. The serum levels of ALT and AST were within normal ranges. Thus, there was no evidence of liver toxicity within the first 2 weeks after treatment. Clearly, toxicity questions must be addressed further in additional longer term studies. 


\section{ACKNOWLEDGEMENT}

This work was supported by grants from the NIDDK (DK58327 and DK067489-JT), the NHLBI (HL59412 and HL69877), and the Alpha One Foundation.

1. Crystal RG. The alpha 1-antitrypsin gene and its deficiency states. Trends Genet 1989;5:411-417.

2. Crystal RG. Alpha 1-antitrypsin deficiency, emphysema, and liver disease. Genetic basis and strategies for therapy. J Clin Invest 1990;85:1343-1352.

3. Birrer P, McElvaney NG, Chang-Stroman LM, et al. Alpha 1-antitrypsin deficiency and liver disease. J Inherit Metab Dis 1991;14:512-525.

4. Perlmutter DH. Alpha-1-antitrypsin deficiency: biochemistry and clinical manifestations. Ann Med 1996;28:385-394.

5. Teckman $\mathrm{JH}, \mathrm{Qu} \mathrm{D}$, Perlmutter $\mathrm{DH}$. Molecular pathogenesis of liver disease in alpha1-antitrypsin deficiency. Hepatology 1996;24:1504-1516.

6. Geller SA, Nichols WS, Dycaico MJ, et al. Histopathology of alpha 1 -antitrypsin liver disease in a transgenic mouse model. Hepatology 1990;12:40-47.

7. Lomas DA, Evans DL, Stone SR, et al. Effect of the $Z$ mutation on the physical and inhibitory properties of alpha 1-antitrypsin. Biochemistry 1993;32:500-508.

8. Lomas DA. Loop-sheet polymerization: the structural basis of $Z$ alpha 1-antitrypsin accumulation in the liver. Clin Sci Lond 1994;86:489-495.

9. Lomas DA. Loop-sheet polymerization: the mechanism of alpha1antitrypsin deficiency. Respir Med 2000;94(Suppl C):S3-S6.

10. Lomas DA, Mahadeva R. Alpha1-antitrypsin polymerization and the serpinopathies: pathobiology and prospects for therapy. J Clin Invest 2002;110:1585-1590.

11. Lomas DA, Lourbakos A, Cumming SA, et al. Hypersensitive mousetraps, alpha1-antitrypsin deficiency and dementia. Biochem Soc Trans 2002;30:89-92.

12. Lomas DA, Parfrey H. Alpha1-antitrypsin deficiency, 4: molecular pathophysiology. Thorax 2004;59:529-535.

13. Lomas DA, Belorgey D, Mallya $M$, et al. Polymerisation underlies alpha1-antitrypsin deficiency, dementia and other serpinopathies. Front Biosci 2004;9:2873-2891.

14. Lomas DA. Molecular mousetraps, alpha1-antitrypsin deficiency and the serpinopathies. Clin Med 2005;5:249-257.

15. Carrell RW, Lomas DA. Alpha1-antitrypsin deficiency - a model for conformational diseases. N Engl J Med 2002;346:45-53.

16. Song $S$, Morgan M, Ellis $T$, et al. Sustained secretion of human alpha1-antitrypsin from murine muscle transduced with adeno-associated virus vectors. Proc Natl Acad Sci USA 1998;95:14384-14388.

17. Song S, Embury J, Laipis PJ, et al. Stable therapeutic serum levels of human alpha-1 antitrypsin AAT; after portal vein injection of recombinant adeno-associated virus rAAV; vectors. Gene Therapy 2001;8:1299-1306.

18. Song $\mathrm{S}$, Scott-Jorgensen $\mathrm{M}$, Wang J, et al. Intramuscular administration of recombinant adeno-associated virus 2 alpha-1 antitrypsin rAAVSERPINA1; vectors in a nonhuman primate model: safety and immunologic aspects. Mol Ther 2002;6:329-335.

19. Conlon TJ, Cossette T, Erger K, et al. Efficient hepatic delivery and expression from a recombinant adeno-associated virus 8 pseudotyped alpha1-antitrypsin vector. Mol Ther 2005;12:867-875.

20. Lu Y, Choi YK, Campbell-Thompson M, et al. Therapeutic level of functional human alpha 1 antitrypsin hAAT; secreted from murine muscle transduced by adeno-associated virus rAAV1; vector. J Gene Med 2006;8:730-735.

21. Flotte TR, Brantly ML, Spencer LT, et al. Phase I trial of intramuscular injection of a recombinant adeno-associated virus alpha 1-antitrypsin rAAV2-CB-hAAT; gene vector to AAT-deficient adults. Hum Gene The 2004;15:93-128.

22. Brantly $M L$, Spencer $L T$, Humphries $M$, et al. Phase I trial of intramuscular injection of a recombinant adeno-associated virus serotype 2 alpha1;antitrypsin AAT; vector in AAT-deficient adults. Hum Gene Ther 2006;17:1177-1186.
23. Zern MA, Ozaki I, Duan L, et al. A novel SV40-based vector successfully transduces and expresses an alpha 1-antitrypsin ribozyme in a human hepatoma-derived cell line. Gene Therapy 1999;6:114-120.

24. Xiao W, Berta SC, Lu MM, et al. Adeno-associated virus as a vector for liver-directed gene therapy. J Virol 1998;72:10222-10226.

25. Chen SJ, Tazelaar J, Moscioni AD, et al. In vivo selection of hepatocytes transduced with adeno-associated viral vectors. Mol Ther 2000;1: 414-422.

26. Gao G, Vandenberghe LH, Wilson JM. New recombinant serotypes of AAV vectors. Curr Gene Ther 2005;5:285-297.

27. Gao GP, Lu Y, Sun X, et al. High-level transgene expression in nonhuman primate liver with novel adeno-associated virus serotypes containing self-complementary genomes. J Virol 2006;80:6192-6194.

28. Grimm D, Pandey K, Nakai HS, et al. Liver transduction with recombinant adeno-associated virus is primarily restricted by capsid serotype not vector genotype. J Virol 2006;80:426-439.

29. Elbashir SM, Martinez J, Patkaniowska A, et al. Functional anatomy of siRNAs for mediating efficient RNAi in Drosophila melanogaster embryo lysate. EMBO J 2001;20:6877-6888.

30. Elbashir SM, Harborth J, Lendeckel W, et al. Duplexes of 21-nucleotide RNAs mediate RNA interference in cultured mammalian cells. Nature 2001;411:494-498.

31. Elbashir SM, Lendeckel W, Tuschl T. RNA interference is mediated by 21- and 22-nucleotide RNAs. Genes Dev 2001;15:188-200.

32. Zolotukhin S, Potter M, Zolotukhin I, et al. Production and purification of serotype 1,2, and 5 recombinant adeno-associated viral vectors. Methods 2002;28:158-167.

33. Loiler SA, Conlon TJ, Song S, et al. Targeting recombinant adenoassociated virus vectors to enhance gene transfer to pancreatic islets and liver. Gene Therapy 2003;10:1551-1558.

34. Carlson JA, Rogers BB, Sifers RN, et al. Multiple tissues express alpha 1-antitrypsin in transgenic mice and man. J Clin Invest 1988;82: 26-36.

35. Carlson JA, Rogers BB, Sifers RN, et al. Accumulation of PiZ alpha 1antitrypsin causes liver damage in transgenic mice. J Clin Invest 1989;83:1183-1190.

36. Dycaico MJ, Grant SG, Felts K, et al. Neonatal hepatitis induced by alpha 1-antitrypsin: a transgenic mouse model. Science 1988;242:1409-1412.

37. Dycaico MJ, Felts K, Nichols SW, et al. Neonatal growth delay in alpha1 -antitrypsin disease. Influence of genetic background. Mol Biol Med 1989;6:137-141.

38. An JK, Blomenkamp K, Lindblad D, et al. Quantitative isolation of alphalAT mutant $Z$ protein polymers from human and mouse livers and the effect of heat. Hepatology 2005;41:160-167.

39. Rudnick DA, Liao Y, An JK, et al. Analyses of hepatocellular proliferation in a mouse model of alpha-1-antitrypsin deficiency. Hepatology 2004:39:1048-1055.

40. Rudnick DA, Shikapwashya O, Blomenkamp K, et al. Indomethacin increases liver damage in a murine model of liver injury from alpha1-antitrypsin deficiency. Hepatology 2006;44:976-982.

41. Gao GP, Alvira MR, Wang L, et al. Novel adeno-associated viruses from rhesus monkeys as vectors for human gene therapy. Proc Natl Acad Sci USA 2002;99:11854-11859.

42. Grimm D, Zhou S, Nakai $\mathrm{H}$, et al. Preclinical in vivo evaluation of pseudotyped adeno-associated virus vectors for liver gene therapy. Blood 2003;102:2412-2419.

43. Miao $\mathrm{CH}$, Nakai $\mathrm{H}$, Thompson AR, et al. Nonrandom transduction of recombinant adeno-associated virus vectors in mouse hepatocytes in vivo: cell cycling does not influence hepatocyte transduction. J Virol 2000;74:3793-3803.

44. Nakai $\mathrm{H}$, Thomas $\mathrm{CE}$, Storm TA, et al. A limited number of transducible hepatocytes restricts a wide-range linear vector dose response in recombinant adeno-associated virus-mediated liver transduction. J Virol 2002:76:11343-11349.

45. Grimm $\mathrm{D}$, Streetz $\mathrm{KL}$, Jopling $\mathrm{CL}$, et al. Fatality in mice due to oversaturation of cellular microRNA/short hairpin RNA pathways. Nature 2006;441:537-541. 